\title{
Explant Cultures From Prostate Needle Biopsies as A Model to Obtain Highly Enriched Populations of Benign and Carcinoma-Associated Fibroblasts
}

Javier Cerda-Infante

Pontifical Catholic University of Chile

Marianela Sánchez

Pontifical Catholic University of Chile

Pablo A. Rojas

Pontifical Catholic University of Chile

Alejandro H. Corvalán

Pontifical Catholic University of Chile

Gonzalo P. Méndez

Pontifical Catholic University of Chile

Bruno Nervi

Pontifical Catholic University of Chile

Gary J. Smith

Roswell Park Cancer Institute

Ignacio F. San Francisco

Pontifical Catholic University of Chile

Alejandro S. Godoy

San Sebastián University

Viviana P. Montecinos ( $\square$ viviana.montecinos@uc.cl)

Pontifical Catholic University of Chile

\section{Research Article}

Keywords: Prostate cancer, Fibroblasts, HPSCs, CAF

Posted Date: March 1st, 2021

DOI: https://doi.org/10.21203/rs.3.rs-181258/v1

License: (1) (1) This work is licensed under a Creative Commons Attribution 4.0 International License.

Read Full License 


\section{Abstract}

Prostate cancer ( $\mathrm{PCa}$ ) is a multifocal neoplasm in which different cancer foci can be found within a single organ. This feature represents a technical difficulty when obtaining highly-enriched primary cultures of human prostate stroma cells (HPSCs), since their isolation involves enzymatic digestion of large pieces of tissue, resulting in an unknown proportion of benign- (BAF) and carcinoma-associated fibroblasts (CAF). Here we report an efficient tissue explant methodology to obtain highly-enriched populations of BAFs or CAFs from benign and malignant human prostate specimens, including needle biopsies. Also, we investigated whether different histological/pathological origins of the prostate tissue determine the activation of distinct transcription programs in HPSCs. Analysis of gene expression profiles revealed that CAFs exhibited a unique transcriptional program characterized by up-regulation of genes involved in proliferation, migration and cell adhesion. CAFs, but not BAFs, expressed typical activation markers such as a-smooth muscle actin, tenascin $\mathrm{C}$ and collagen 1 and stimulated tumor growth of PCa cells in vivo. Our study indicated that gene expression profile of HPSCs was determined mostly by the zone rather than the pathological state of the prostate tissue and that tissue explants from needle biopsies provide a valuable tool to obtain highly enriched primary cultures of HPSCs.

\section{Introduction}

Prostatitis, benign prostatic hyperplasia (BPH) and prostate cancer ( $\mathrm{PCa}$ ) share common alterations in their functional interaction between the glandular epithelium and the stromal cells that result in altered expression of chemokines, cytokines, matrix remodeling factors, and chronic inflammatory processes. These changes determine the formation of a "reactive stroma" that shares common features with the activated stroma present in the wound healing process[1-5]. In the benign adult prostate, stromal and epithelial cells synthesize and respond to growth factors in a reciprocal and dynamic manner to maintain the normal tissue structure and functionality $[3,6,7]$. Paracrine growth factors produced by the stroma cells provide regulatory signals to maintain the homeostasis and differentiated state of the epithelium. In addition, the stroma plays a key role in the development and progression of several human prostatic diseases[1-5].

The human prostate comprises three histologically and anatomically distinct zones; the peripheral zone (PZ), the central zone (CZ) and the transitional zone (TZ)[3]. BPH occurs almost exclusively within the transitional zone of the prostate, while PCa occurs primarily in the peripheral zone [3]. Gene expression profile analyses of cells from these zones in normal prostate revealed differences in gene expression patterns between the peripheral and transition zones that could modulate stromal-epithelial cell interaction [1]. In addition, BPH appears associated with a state of hyperplasia of both the stromal and epithelial compartments [2, 3], suggesting that prostate disease susceptibility is zone-dependent and involves alterations of both, the stromal and the epithelial compartments. Despite these suggesting evidences, little is known about the molecular profiles of stromal cells within the different compartments of the prostate, or during different disease states. 
The ability to obtain stroma cell cultures from human prostate tissue (HPSCs), in a reliable and consistent manner, represents an invaluable tool for the study of the biology of human prostate tissue under normal and pathological conditions. Moreover, it could provide insight into the molecular changes that occur in the microenvironment during disease development and progression, including BPH and PCa. $\mathrm{BPH}$ and PCa are highly heterogeneous diseases. PCa is a multifocal neoplasm in which several different cancer foci can be found within a single human malignant prostate, with foci in close proximity to benign areas. This feature represents a technical problem difficult to overcome since the standard procedures for isolation of primary stromal cell cultures (HPSCs) involves enzymatic digestion of large pieces of tissue, usually over a gram, resulting in primary cultures that contain an unknown proportion of benign (BAF) and carcinoma-associated fibroblasts (CAF) [11]. Furthermore, procurement of fresh prostate tissue specimens remains a challenge, and specimens generally are restricted to radical prostatectomy (RP) tissue remnants and transitional zone specimens from benign hyperplasia resections of patients undergoing surgery to relieve blockage. To our knowledge, our study demonstrates for the first time that prostate tissue explants can be routinely established from needle biopsies to allow a rapid harvesting of primary cultures of human stromal cells from the small fresh tissue specimens without disrupting pathology analysis and facilitating sampling of specific human prostate zones undergoing diverse pathologies, Gleason grades and stages.

\section{Materials And Methods}

\section{Samples Collection}

Human prostate tissue was obtained from needle biopsies surgically resected from 108 different patients at the Clinical Hospital from Pontificia Universidad Católica de Chile (PUC). Informed written consent was obtained from all participants, and all protocols were approved by the Ethical committee of PUC. Benign prostate needle core specimens from the transitional zone (TZ-BAF) were obtained from patients with $\mathrm{BPH}$ that underwent holmium laser enucleation of the prostate HOLEP (18 samples) or transurethral resection of the prostate TURP (23 samples). Peripheral zone needle biopsies from benign (PZ-BAF) and malignant human prostate tissue (PZ-CAF) were obtained from radical prostatectomy tissue remnants or needle biopsies from PCa patients (45 samples), respectively. H\&E staining of formalin-fixed, paraffinembedded (FFPE) tissue sections were prepared from each specimen to confirm presence of benign or malignant human prostate tissue and to obtain information about the histological subtype and histopathological grade through the Department of Pathology at PUC. All experiments were performed in accordance with relevant guidelines and regulations for human studies.

\section{Tissue explants technique}

Primary cultures of HPSCs were established from histologically confirmed benign, BPH or PCa tissues by the explant culture technique. Briefly, prostate tissue specimens (needle biopsies, HOLEP and TURP) were cut into 6-8 pieces of approximately $1 \times 2 \mathrm{~mm}$ each and placed on $60 \mathrm{~mm}$ Petri dishes, containing DMEM medium (Gibco BRL, Life Technologies) supplemented with 10\% fetal bovine serum (Hyclone 
Laboratories, Logan, Ut), $2 \mathrm{mM}$ glutamine, $100 \mathrm{U} / \mathrm{ml}$ penicillin, and $0.1 \mathrm{mg} / \mathrm{ml}$ streptomycin (all from Hyclone Laboratories). Tissue explants were maintained in $60 \mathrm{~mm}$ Petri dishes at $37^{\circ} \mathrm{C}$ and $5 \% \mathrm{CO}_{2}$ humidified incubator. The medium was replaced every three days. The cell sprouting occurred within 5-12 days. The purity of stromal cell cultures was estimated by staining the primary cultures with an antibody against epithelial pan-cytokeratin.

\section{Cell growth}

HPSCs were placed on 96 multi-well dish and cultured with DMEM medium supplemented with $10 \%$ FBS for $1,3,5,7,10$, and 15 days. Attached cells were collected by trypsinization and counted using a hemocytometer. Trypan blue dye exclusion was used to determine viable cells. Doubling time (Dt) was calculated as the log of the ratio of the final count $(\mathrm{N})$ to the starting (baseline) count $(\mathrm{Xo})$, divided by the $\log 2$; that is $\mathrm{Dt}:[\log (\mathrm{N} / \mathrm{Xo})] / \log 2$.

\section{Microarray analysis}

Total RNA from HPSCs was isolated using Trlzol solution (Life TechnologiesTM) according to manufacturer's instructions. The Ambion MessageAmp II-Biotin Enhanced kit was used to synthesize double-stranded cDNA and produce biotin-labeled cRNA from $500 \mathrm{ng}$ of total RNA. After fragmentation, $10 \mathrm{mg}$ of cRNA were hybridized at $45^{\circ} \mathrm{C}$ for $16 \mathrm{~h}$ to Illumina HT12v4 BeadChips oligonucleotide arrays containing probes to $>47,000$ transcripts. The bioinformatics analyses were performed using the software TMEV v4.7.4.

\section{Xenograft experiments}

In vivo experiments were conducted in accordance with the ARRIVE (Animal Research: Reporting of In Vivo Experiments) guidelines following the ethical approval (bioethical committee of PUC) and, relevant guidelines. 8-week-old male NOD.Cg-Prkdc ${ }^{\text {scid }} \| 2 \mathrm{rg}^{\mathrm{tm} 1 \mathrm{Wjl}} / \mathrm{SzJ}$ mice (The Jackson Laboratory) were injected subcutaneously with $1 \times 10^{6} \mathrm{PC} 3$ cells in the right lateral flanks. For cell recombination analyses, $1 \times 10^{6}$ PC3 cells and $2 \times 10^{6}$ fibroblasts, isolated from PZ-BAF or PZ-CAF, were co-injected. Animals were monitored daily and tumor size was measured every 3 days using a caliper. Tumor volumes were determined using the formula: width ${ }^{2} \mathrm{x}$ length $\mathrm{x} \pi / 6$.

\section{Statistical analysis}

Data is presented as means \pm SD from at least three independent experiments. Statistical significance was calculated using Student's t-test. In each case, a $p$-value $<0.01$ was considered to represent statistically significant differences.

\section{Results}


One hundred and eight patients that underwent either prostate surgery or needle biopsy were selected for this study. The clinical and pathological features of the patient cohort are shown in Table 1. Fresh tissue specimens obtained from surgical procedures (TURP or HOLEP) or from ultrasound needle biopsies were used for the propagation of primary cultures of stromal cells (HPSCs) using the tissue explant technique (Fig. 1). We established HPSCs primary cultures from a total of 67 trans-rectal needle biopsies obtained from separate individuals or from surgical specimens; 23 samples from TURP and 18 samples from HOLEP (Table 1). Prostate tissue explants were evenly distributed over the surface of tissue culturetreated dishes in a minimal volume of culture medium, to preserve the tissue in contact with the culture surface (Fig. 1C). This procedure allowed maintaining the adherence of the tissue wedges in close to $100 \%$ of the samples analyzed (Fig. 1C). After 7-10 days of explant culture, spindle-shaped cells started to migrate out of tissue explants onto the culture dish (Fig. 1C, 1E). Significant differences were observed in the average of sprouting days between the different samples. HPSCs obtained from benign transition zone using the TURP procedure took longer to start to migrate out of the explanted tissue (TZ-BAF-T; 23 days) when compared to HPSCs obtained from the HOLEP procedure (TZ-BAF-H, average 16 days) (Fig. 1E). On the other hand, in tissue samples obtained from peripheral zone through needle biopsies (PZ-CAF and PZ-BAF), the average time for the sprouting process to start was 10 days or less. HPSCs from PCa patients (PZ-CAF) took the shortest time to sprout out the explanted tissue of all groups (7 days). Statistically significant differences in the average sprouting time were found in PZ-CAF when compared to PZ-BAF. The efficiency (percentage) of the explant technique (i.e. the percentage of wedges that sprouted cells compared from the total that adhered to the culture dish) was nearly $97 \%$ in the tissue samples obtained through needle biopsies (22 in PZ-BAF and 45 in PZ-CAF) (Fig. 1F). However, from the tissue samples obtained through surgical procedures, 79\% of HOLEP (TZ-BAF-H) and $62 \%$ of TURP (TZBAF-T) specimens sprouted out, which indicated that the efficiency of the explant technique was significantly higher when samples were obtained from needle biopsies (94\% PZ-BAF and 97\% PZ-CAF). Samples obtained from TURP showed the lowest efficiency of all procedures (Fig. 1F). 
Table 1

Clinicopathologic characteristics of the patient's cohort

\begin{tabular}{|c|c|c|c|c|}
\hline Procedure & $\begin{array}{l}\text { HOLEP } \\
(n=18)\end{array}$ & $\begin{array}{l}\text { TURP } \\
(n=23)\end{array}$ & Biopsy Pre-Qx $(n=22)$ & $\begin{array}{l}\text { Biopsy Pre-Qx/RP } \\
(n=45)\end{array}$ \\
\hline Diagnosis & $\mathrm{BPH}$ & $\mathrm{BPH}$ & without neoplasia & $\mathrm{PCa}$ \\
\hline Type of sample & TZ-BAF-H & TZ-BAF-T & PZ-BAF & PZ-CAF \\
\hline Age (yr.) & $54-77$ & $55-84$ & $52-74$ & $41-85$ \\
\hline Prostate volume (cc) & $33-133$ & $39-74$ & $23-97$ & $27-200$ \\
\hline PSA (ng/ml) & $0.8-21.3$ & $0.5-75$ & $3.2-8.7$ & $3.3-4730$ \\
\hline Gleason & $\mathrm{N} / \mathrm{A}$ & $\mathrm{N} / \mathrm{A}$ & $\mathrm{N} / \mathrm{A}$ & $6.0-9.0$ \\
\hline Metastasis & $\mathrm{N} / \mathrm{A}$ & $\mathrm{N} / \mathrm{A}$ & $\mathrm{N} / \mathrm{A}$ & M0-M1 \\
\hline \multicolumn{5}{|c|}{$\begin{array}{l}\text { TZ: transition zone; PZ: peripheral zone; BAF: benign-associated fibroblast; CAF: carcinoma- } \\
\text { associated fibroblast; BPH: benign prostatic hyperplasia; CaP: prostate cancer; HOLEP: holmium lase } \\
\text { enucleation of the prostate; TURP: transurethral resection of the prostate; RP: radical prostatectomy; } \\
\text { N/A: not applicable. }\end{array}$} \\
\hline
\end{tabular}

An immunohistochemical characterization of the HPSC cells isolated from human prostate tissue specimens revealed that, even when most migrating cells possessed a typical mesenchymal morphology, there was a mix of epithelial and stromal cells in all groups analyzed, as determined by expression of the epithelial and mesenchymal markers cytokeratin and vimentin, respectively (Fig. 2A, B). A detailed immunostaining analysis for the expression of vimentin, aSMA and pan-cytokeratin was performed for PZ-BAF and PZ-CAF at different cell culture passages (from passage 0 to passage 4, Fig. 2C, D). Data indicated that for benign and malignant primary cultures of peripheral zone at passages 0 and 1, a small proportion of cells were positive for cytokeratin (Fig. 2C, D). However, at passage 3, the primary cultures were positive only for stroma markers, indicating that from passage 3 and on, primary cultures isolated from prostate needle biopsies were composed mostly by fibroblasts without evident epithelial cell contamination (Fig. 2C, D). In summary, the needle biopsies explant technique allowed us to obtain highly enriched cultures of BAF from the peripheral zone, and CAF from either, the primary tumor or locally advanced/metastatic PCa.

The morphological characteristics and proliferative rate of TZ-BAF, PZ-BAF and PZ-CAF cultures were evaluated in vitro using growth curve and Ki-67 expression analyses. Fibroblasts maintained their phenotypic characteristics during culture showing a uniform spindle-shaped morphology (Fig. 3A). Quantitation of cell growth was performed using the trypan blue exclusion method. Our results indicated that primary cultures of fibroblasts obtained from explants of malignant PZ tissue showed higher level of proliferation compared to both, benign TZ or PZ tissues (Fig. 3B). Based on the data presented in Fig. 3B, we determined the doubling time (Dt) for each group. Our results indicated doubling times of 3.1, 7.9 and 8.7 days for PZ-CAF, TZ-BAF and PZ-BAF, respectively. No significant difference in doubling time were observed between TZ-BAF and PZ-BAF $(p<0.05)$. To confirm the differences found between CAF and BAF, 
we performed an immunofluorescence analysis for the expression of the cell proliferation marker, Ki- 67. Percentages of Ki-67-positive cells were significantly higher in PZ-CAF ( $80 \%)$ compared to both, TZ-BAF and PZ-BAF (less than $20 \%$ for both groups, Fig. 3C, D) primary cultures. Based on these observations, we hypothesized that prostatic fibroblast cultures of different histological and pathological origins have distinct transcriptional profiles. To test this hypothesis, we performed a transcriptomic analysis of a pool of $10 \mathrm{cDNA}$ samples from each origin (TZ-BAF, PZ-BAF and PZ-CAF) using an Illumina Platform array technology. Prostate fibroblasts samples were analyzed by hierarchical clustering[13] using 18,392 genes, or considering only the 393 genes differentially expressed (Fig. 3E). Dendrogram analysis (Fig. 3E) showed that TZ-BAF cultures clustered differentially from both, PZ-BAF and PZ-CAF, which indicates that the most striking difference of the transcriptional programs was associated with the histological origin of the fibroblasts (transitional vs peripheral zones). Heatmap analysis of the genes expressed 10 -fold higher or 10-fold lower (393 in total) in each zone is showed in Fig. 3F. Figure $3 \mathrm{G}$ and $3 \mathrm{H}$ represents the genes that are most differentially expressed in benign PZ fibroblasts compared to benign TZ fibroblasts. Gene Ontology (GO) analyses revealed that many of those genes were involved in biological processes that are known to be important in the development of benign prostatic diseases, such as smooth muscle contraction, cytoskeleton organization, lipid metabolic process and immune response. Since PCa originates mostly in the peripheral zone, the comparison of the transcriptional profiles of fibroblasts obtained from benign or malignant PZ tissues revealed a set of differentially expresses genes (Fig. 3G, $\mathrm{H})$. GO analyses indicated that many of those genes were involved in processes that are associated with the activation of biological programs such as transcription, inflammatory response, cell adhesion and motility. To confirm the gene expression changes observed by microarray analyses, real-time RT-PCR and western blot were performed in TZ-BAF, PZ-BAF and PZ-CAF for the typical activated markers genes associated to CAF. qRT-PCR analyses indicated that the activation markers a-smooth muscle actin (aSMA), tenascin C (TNC) and collagen 1 (Col1), were significantly up-regulated in CAF compared to BAF (Fig. 4A). No significant changes were observed for vimentin and fibroblast activation proteins (FAP; Fig. 4A). Western blot analyses of the smooth muscle cell markers calponin and aSMA, and the fibroblast marker vimentin, revealed that TZ-BAF cultures were heterogeneous and composed mostly of smooth muscle cells (calponin positive), while PZ-BAF cultures exhibited less expression of the smooth muscle marker calponin (Fig. 4B, C). In contrast, PZ-CAF cultures showed a phenotypic switch to a predominantly myofibroblast population, a typical feature of CAF (Fig. 4B, C).

As studied extensively, CAF showed an increased ability to affect tumor progression in initiated epithelial cells compared to BAF $[4,14]$. Therefore, we decided to test whether our CAF cultures, obtained from needle biopsies, could increase tumor formation and growth in vivo. The PCa cell line (PC3) was grafted alone, or in combination with PZ-BAF or PZ-CAF, subcutaneously in the flank of immunocompromised mice. After 30 days, tissue recombinants composed of PC3-CAF tumors exhibited striking growth with a maximum wet weight of $2942 \mathrm{mg}$, and an average weight of $2118 \mathrm{mg}$. In contrast, PC3-BAF tissue recombinants, and PC3 alone, demonstrated low growth after 30 days of the co-engraftment. PC3-BAF showed a maximum wet weight of $1080 \mathrm{mg}$, and an average weight of 738. PC3 alone showed a maximum wet weight of $1087 \mathrm{mg}$, and an average weight of $765 \mathrm{mg}$ (Fig. 4C, D). These results confirmed 
that the tissue explant procedure from needle biopsies allowed establishment of CAF cultures that expressed typical activation markers and stimulated tumor growth of PCa cells in vivo.

\section{Discussion}

Unraveling the role of fibroblasts in cancer progression, response to therapy and acquisition of recurrence remain crucial to understanding the contribution of the tumor microenvironment to prostate cancer biology. In solid tumors, it has been demonstrated that paracrine communication between CAF and cancer cells is regulated by a reciprocal set of signals/factors that promote tumor growth, cancer cell invasion and metastasis by enhancing the survival, proliferation, stemness, metastasis-initiating capacity of cancer cells, and resistance to therapy, among other properties[2, 15-20]. In PCa, it has been demonstrated that CAFs define the evolution of the castration-resistant phenotype during PCa progression[17]. Kato et. al., using high and low passages CAF as a model to study the heterogeneity of tumor associated fibroblasts demonstrated that the CD105 + CAF population mediated prostatic tumor epithelial cell differentiation and castration resistance in a paracrine manner[17]. Due to the multifocal nature of $\mathrm{PCa}$, obtaining pure cancer areas from radical prostatectomy tissue specimens for in vitro and preclinical research remains a challenge. Furthermore, the use of radical prostatectomy samples limits primary stroma cell culture mostly to better-differentiated cancers since the majority of patients with high grade PCa tumors with nodal metastases usually are inoperable. Needle biopsies provide an alternative source of high-grade malignant PCa tissue samples for procurement of tissue from patients before systemic androgen ablation to provide cells with unique biological behaviors. To our knowledge, this is the first article to report the isolation of stromal cells from needle biopsy specimens. This methodology can be established to allow a rapid harvesting of primary cultures of human stromal cells from the small fresh tissue specimens without disrupting pathology analysis and facilitating sampling of specific human prostate zones undergoing diverse pathologies, Gleason grades and stages. In addition, other types of primary cells, such as the prostate epithelial cells, could be potentially isolated and cultured using this technique, which could improve our understanding of the cell type-specific contribution from the tumor microenvironment to the biology of prostate cancer.

Comparison of the cellular and molecular properties of CAF derived from moderate grade or nonmetastatic PCa versus high-grade metastatic PCa may unravel molecular mechanisms crucial for the understanding of the progression of PCa. Our gene expression analysis indicated that the gene expression profile of fibroblasts was zone-specific, since BAFs obtained from the peripheral zone shared more similarities with CAF (also obtained from peripheral zone) than BAF obtained from the transitional zone. Other studies also have reported differences at the molecular level between the different prostate zones, although these studies were not restricted to the stromal compartment[8, 21, 22]. Divergent hormone responsiveness of primary cultures of PZ- and TZ-derived stem cells[21] as well as differential expression of certain hormone-metabolizing enzymes in the inter-zone (e.g. CYP1B1)[22] have been reported. Van der Heul-Nieuwenhuijsen, et al.,[8] indicated that peripheral and transition zones of human prostate tissue obtained from radical prostatectomy remnant tissue have identical stroma and epithelium distribution and equal expression of cell-type markers, suggesting that the quantity and phenotype of the 
specific cell types is not different between these two prostate zones. Moreover, in this study, microarrays and RT-PCR analyses in human prostate tissue from PCa (PZ) and BPH (TZ) demonstrated 12 differentially expressed zone-specific genes in the peripheral zone of prostate relative to transition zone[8]. However, the contribution of the epithelial or the stromal compartment to the differential expression of these 12 genes was not explored in this study. Together, our and other studies indicated that PZ and TZ of the prostate have differential gene expression patterns for individual cell types that could be associated with the pathological origin of the prostatic diseases. Further studies are necessary to elucidate the specific cellular and molecular mechanisms activated in each zone that modulate the stromal-epithelial cell interaction in the prostate under normal and pathological conditions.

\section{Declarations}

\section{Conflict of interest}

The authors declare no conflict of interest.

\section{Acknowledgments}

Supported by FONDECYT \#1150397 to VPM and CONICYT graduate fellowship to JCl (CONICYTPCHA/Doctorado Nacional/2014/\#21130970). The authors thank the services provided by UC CIBEM Animal Facility (PIA CONICYT*ECM-07).

\section{References}

1. Bechis, S. K., Otsetov, A. G., Ge, R. \& Olumi, A. F. Personalized medicine for the management of benign prostatic hyperplasia.J Urol. 2014 Jul:192:16-23

2. Giannoni, E. et al. Reciprocal activation of prostate cancer cells and cancer-associated fibroblasts stimulates epithelial-mesenchymal transition and cancer stemness.Cancer Res. 2010 Sep1: 70:6945-56

3. Lee, K. L. \& Peehl, D. M. Molecular and cellular pathogenesis of benign prostatic hyperplasia. J Urol. Nov, 1721784-1721791 (2004).

4. Olumi, A. F. et al. Carcinoma-associated fibroblasts direct tumor progression of initiated human prostatic epithelium. Cancer Res. 1, 595002-595011 (1999 Oct).

5. Rowley, D. R. What might a stromal response mean to prostate cancer progression? Cancer Metastasis Rev. 17, 411-419 (1998).

6. Tuxhorn, J. A., Ayala, G. E. \& Rowley, D. R. Reactive stroma in prostate cancer progression. J Urol. Dec, 1662472-1662483 (2001).

7. Tuxhorn, J. A. et al. Reactive stroma in human prostate cancer: induction of myofibroblast phenotype and extracellular matrix remodeling.Clin Cancer Res. 2002 Sep:8:2912-23 
8. van der Heul-Nieuwenhuijsen, L., Hendriksen, P. J., van der Kwast, T. H. \& Jenster, G. Gene expression profiling of the human prostate zones. BJU Int. 2006 Oct: 98:886 - 97

9. Schauer, I. G. \& Rowley, D. R. The functional role of reactive stroma in benign prostatic hyperplasia.Differentiation. 2011 Nov-Dec:82:200-10

10. Strand, D. W., Costa, D. N., Francis, F., Ricke, W. A. \& Roehrborn, C. G. Targeting phenotypic heterogeneity in benign prostatic hyperplasia.Differentiation. 2017 Aug04: 96:49-61

11. Peehl, D. M. Primary cell cultures as models of prostate cancer development.Endocr Relat Cancer. 2005 Mar:12:19-47

12. Takashima, A. Establishment of fibroblast cultures. Curr Protoc Cell Biol. 2001 May: Chap. 2:Unit 21

13. Zhao, F. J., Han, B. M., Yu, S. Q. \& Xia, S. J. Tumor formation of prostate cancer cells influenced by stromal cells from the transitional or peripheral zones of the normal prostate. Asian J Androl. 2009 Mar: 11:176- 82

14. Orimo, A. et al. Stromal fibroblasts present in invasive human breast carcinomas promote tumor growth and angiogenesis through elevated SDF-1/CXCL12 secretion.Cell. 2005 May6:121:335-48

15. Ren, Y. et al. Paracrine and epigenetic control of CAF-induced metastasis: the role of HOTAIR stimulated by TGF-ss1 secretion.Mol Cancer. 2018 Jan11: 17:5

16. Su, S. et al. CD10(+)GPR77(+) Cancer-Associated Fibroblasts Promote Cancer Formation and Chemoresistance by Sustaining Cancer Stemness.Cell. 2018 Feb8: 172:841 - 56 e16

17. Kato, M. et al. Heterogeneous cancer-associated fibroblast population potentiates neuroendocrine differentiation and castrate resistance in a CD105-dependent manner.Oncogene. 2019 Jan:38:71630

18. Kalluri, R. The biology and function of fibroblasts in cancer.Nat Rev Cancer. 2016 Aug23: 16:582-98

19. LeBleu, V. S. \& Kalluri, R. A peek into cancer-associated fibroblasts: origins, functions and translational impact. Dis Model Mech. 19, 11 (2018 Apr).

20. Singh, M. et al. Stromal androgen receptor in prostate development and cancer. Am J Pathol. 2014 Oct: 184:2598 - 607

21. Kirschenbaum, A. et al. Sex steroids have differential effects on growth and gene expression in primary human prostatic epithelial cell cultures derived from the peripheral versus transition zones.Carcinogenesis. 2006 Feb:27:216-24

22. Ragavan, N. et al. CYP1B1 expression in prostate is higher in the peripheral than in the transition zone.Cancer Lett. 2004 Nov8: 215:69-78

\section{Figures}


Figure 1

Cerda-Infante et al.
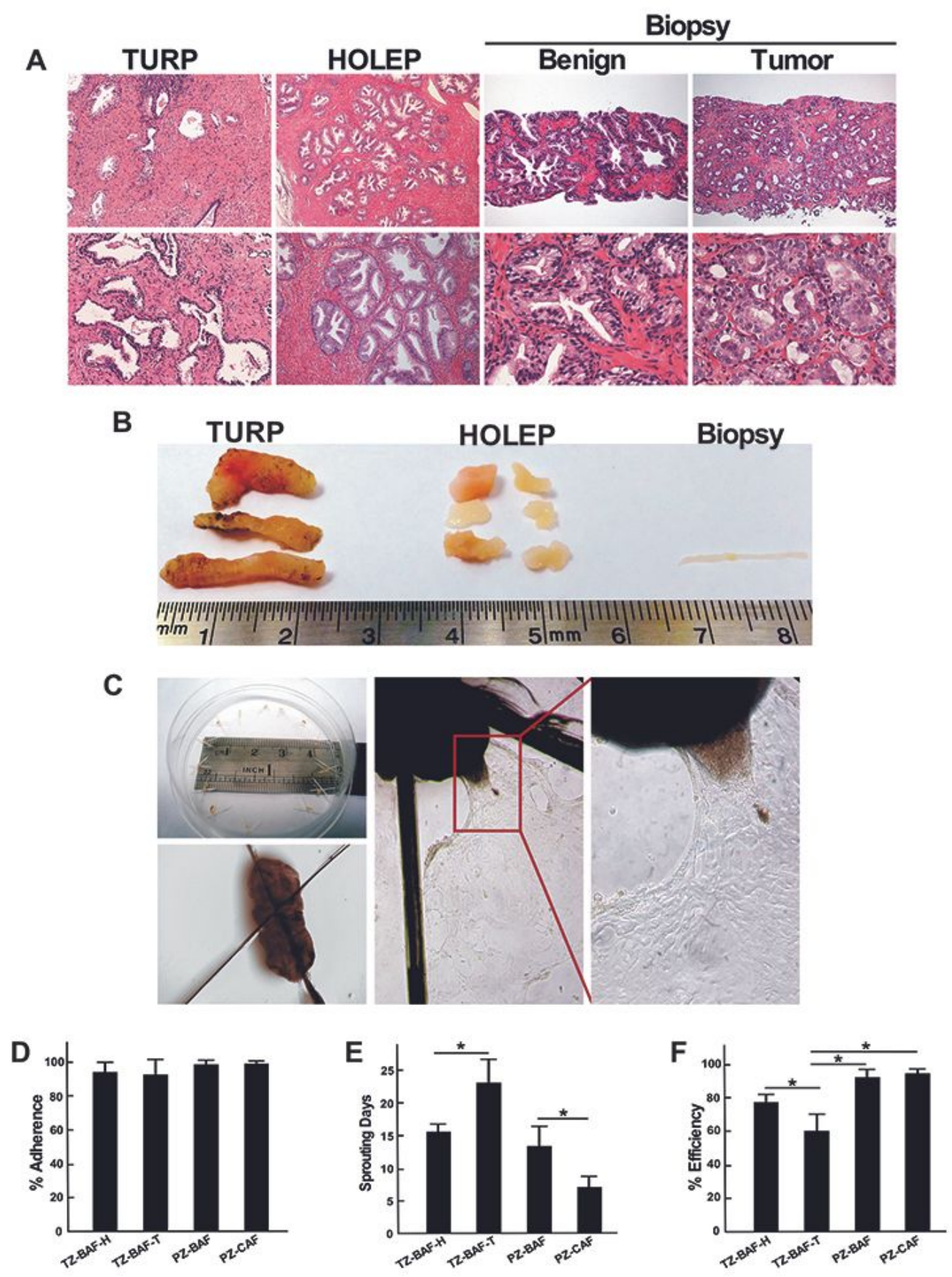

\section{Figure 1}

Establishment and characterization of tissue explants techniques from benign and malignant human prostate tissue. A. H\&E staining of human prostate tissue obtained from TURP-surgery, HOLEP-surgery and needle-biopsy from benign and tumor tissue. Upper pictures: 100x and lower pictures: 200x magnification. B. Representative images of the human prostate tissues obtained from TURP-surgery, HOLEP-surgery, and needle biopsy. C. Left panels, tissue explant culture assay. Middle and right panels, 
phase contrast images. D. Adherence percentage of prostate tissue explants respect to total explants placed. E. Sprouting cells time of prostate tissue explants from TURP (23 days, TZ-BAF-T), HOLEP (16 days, TZ-BAF-H), the benign needle biopsy (13 days, PZ-BAF) and tumor needle biopsy (7 days, PZ-CAF). F. Efficiency of adherence (\%) of prostate tissue explants with respect to total explants seeded. ${ }^{*}<<0,01$.

Figure 2

Cerda-Infante et al.

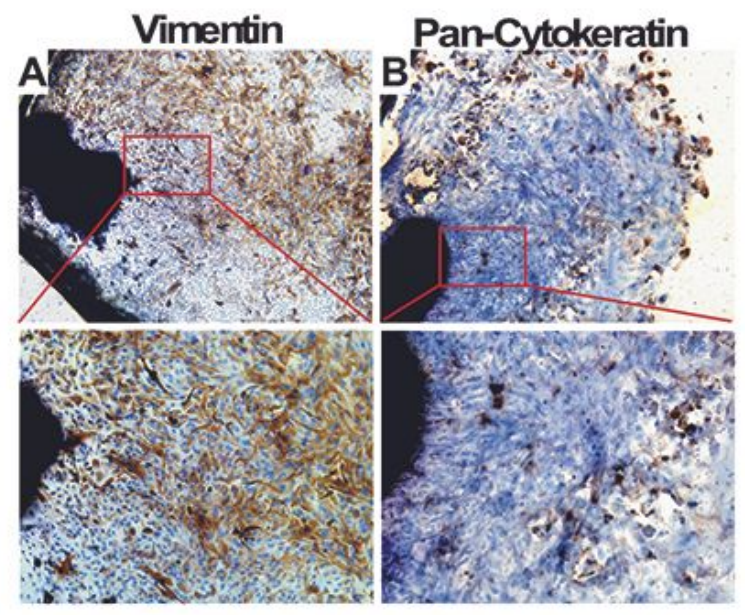

C
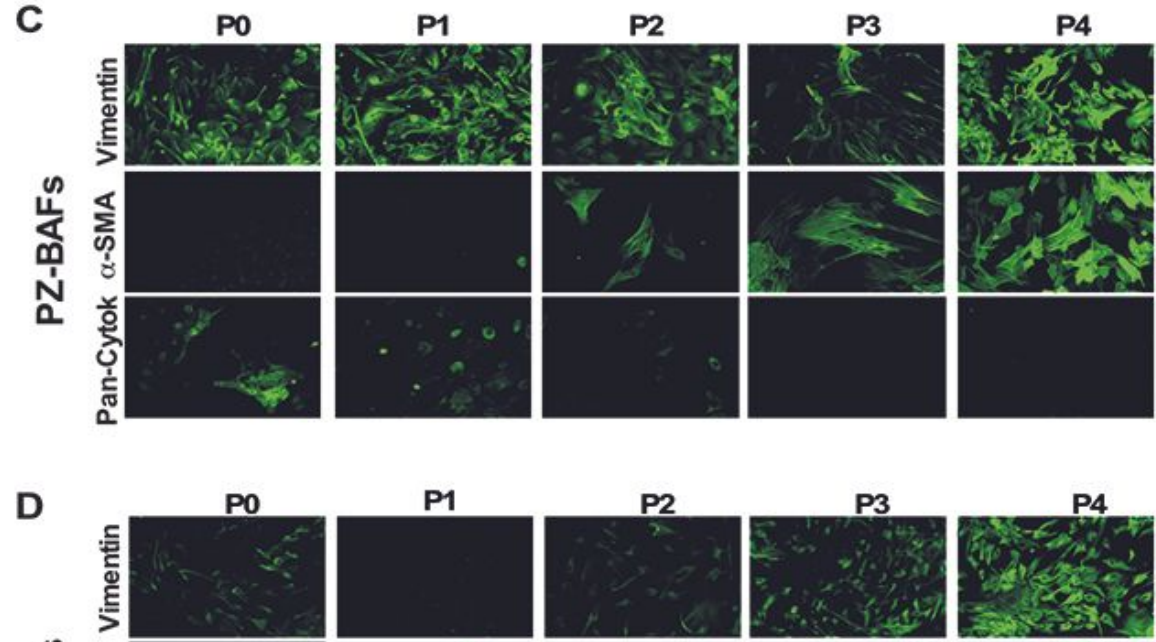

选
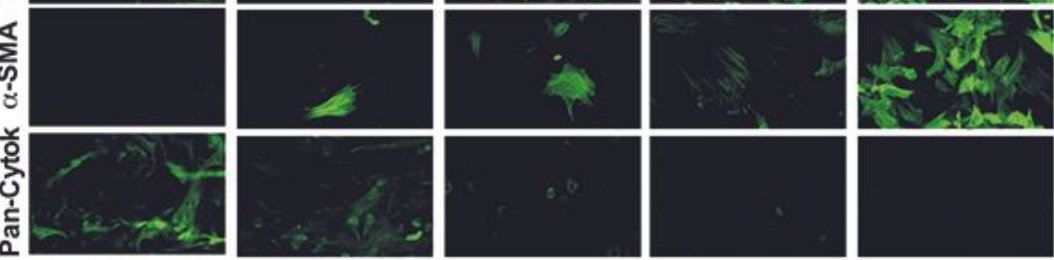

Figure 2 
Immunostaining analysis of stromal and epithelial markers in primary cultures of HPSCs isolated from human prostate tissue explants. A-B. Immunocytochemical analysis of the tissue explant culture for the mesenchymal phenotype markers, vimentin (A), and the epithelial phenotype marker, pan-cytokeratin (B). Magnifications in A and B: upper panels 10X and lower panels 20X. C-D. Immunofluorescence analyses of HPSCs from PZ-BAF (C) and PZ-CAF (D) from passage 0 (P0) to passage 4 (P4) of culture. HPSCs were immunostained for the mesenchymal phenotype markers, vimentin and aSMA, and the epithelial phenotype marker, pan-cytokeratin. Magnifications panels C and D: 20X.

Figure 3

\section{Cerda-Infante et al.}

A

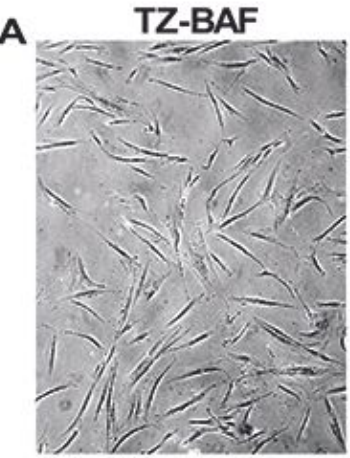

C

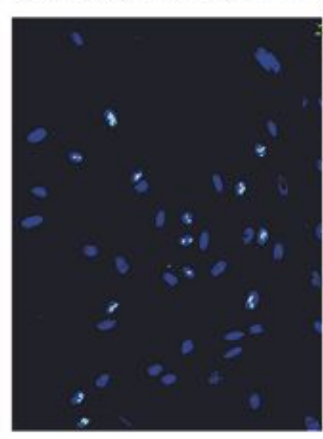

E

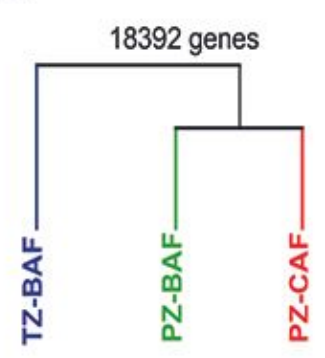

393 genes

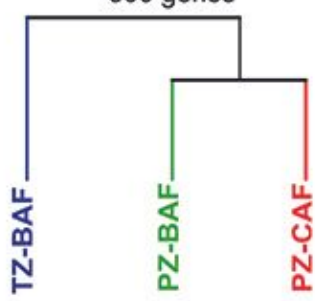

PZ-BAF
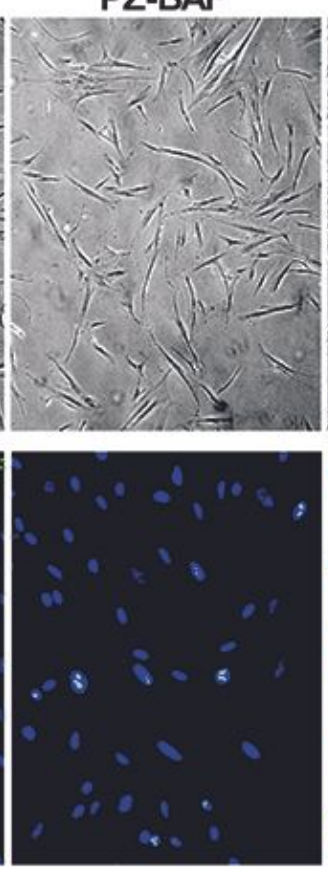

F

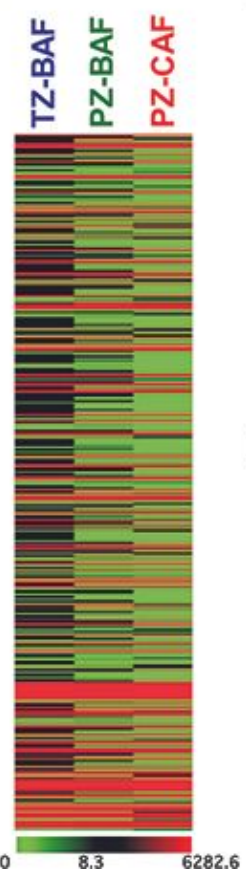

PZ-CAF
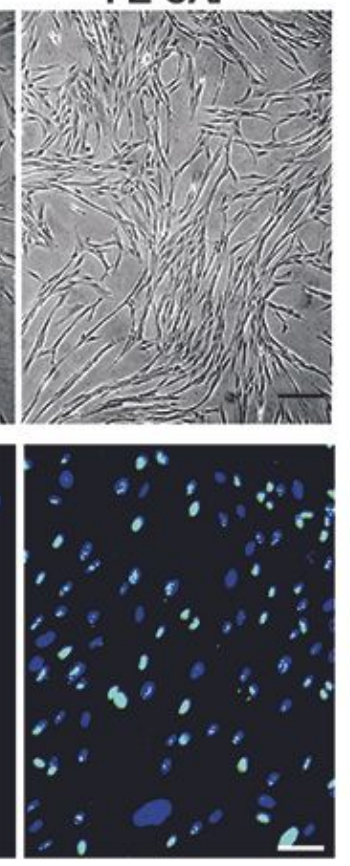

G

\begin{tabular}{|l|c|c|l|}
\hline Gene & PZ-CAF & PZ-BAF & Biological Process \\
\hline GPR116 & 1 & 380 & transcription \\
\hline CST1 & 291.8 & 2.3 & cysteine protease inhibitor activity \\
\hline FOXS1 & 1 & 195,9 & transcription \\
\hline CCDC81 & 1 & 150,1 & protein binding \\
\hline CCL7 & 71 & 1 & inflammatory response \\
\hline NLGN4X & 57.5 & 1 & cell adhesion \\
\hline CLGN & 40.7 & 1 & calcium ion binding \\
\hline CDH10 & 40.2 & 1 & homophilic cell adhesion \\
\hline EPHA3 & 54 & 1.4 & protein amino acid phosphorylation \\
\hline KAL1 & 31 & 1 & cell motility \\
\hline
\end{tabular}

H

\begin{tabular}{|l|c|c|l|}
\hline Gene & PZ-BAF & TZ-BAF & Biological Process \\
\hline MYH11 & 414.1 & 1 & smooth muscle contraction \\
\hline NTF3 & 121.2 & 1 & cell motility \\
\hline CACNA1H & 117 & 1 & calcium ion transport \\
\hline KISS1 & 111.1 & 1 & cytoskeleton organization and biogenesis \\
\hline SPINT2 & 105.2 & 1 & cell motility \\
\hline CX3CL1 & 85.4 & 1 & immune response \\
\hline CD36 & 924.3 & 13.1 & lipid metabolic process \\
\hline PCSK1N & 66.9 & 1 & neuropeptide signaling pathway \\
\hline EFHD1 & 1154.1 & 17.6 & neurite development \\
\hline SCG2 & 60.2 & 1 & angiogenesis \\
\hline
\end{tabular}




\section{Figure 3}

Cell growth, proliferation and gene expression profile assays of HPSCs isolated from benign or malignant tissue. A. Phase contrast images of HPSCs on P4. Black bar: $100 \mu \mathrm{M}$. B. Growth curves of HPSCs isolated from TZ-BAF, PZ-BAF, and PZ-CAF. Doubling times were 7.9 days for TZ-BAF, 8.7 days for PZ-BAF and 3.1 days for PZ-CAF. C. Immunocytochemical analyses of Ki-67 expression in HPSCs. DAPI was used to counterstain nuclei. White bar: $50 \mu \mathrm{m}$. D. Quantitation of Ki-67 positive cells from panel C. ${ }^{*}=p<0,01$. E. Upper panel, dendrogram clustering for total genes analyzed in mRNA microarray. Lower panel, dendrogram analysis of the 393 genes differentially expressed between the three groups. F. Relative expression map of the genes corresponding to the higher and lower expression (393 in total) in each prostate zone. G-H. Relative expression levels of the genes that are most differentially expressed in PZBAF compared to TZ-BAF cultures $(G)$ and PZ-CAF vs PZ-BAF $(H)$. 
Figure 4

Cerda-Infante et al.

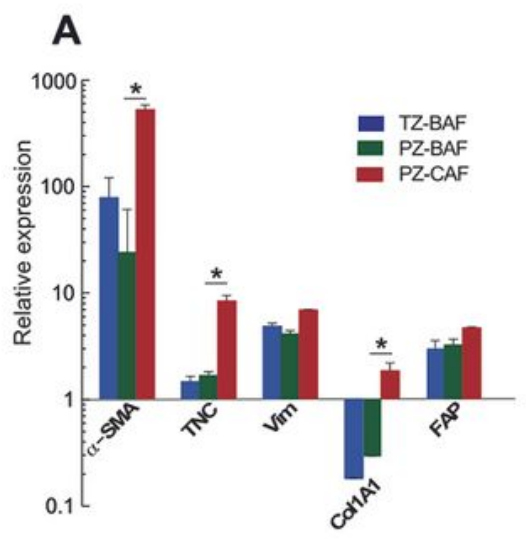

C

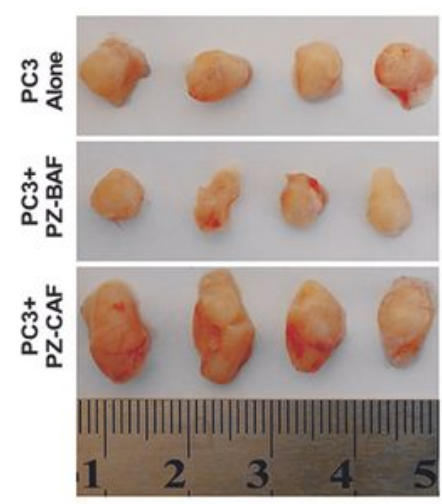

B
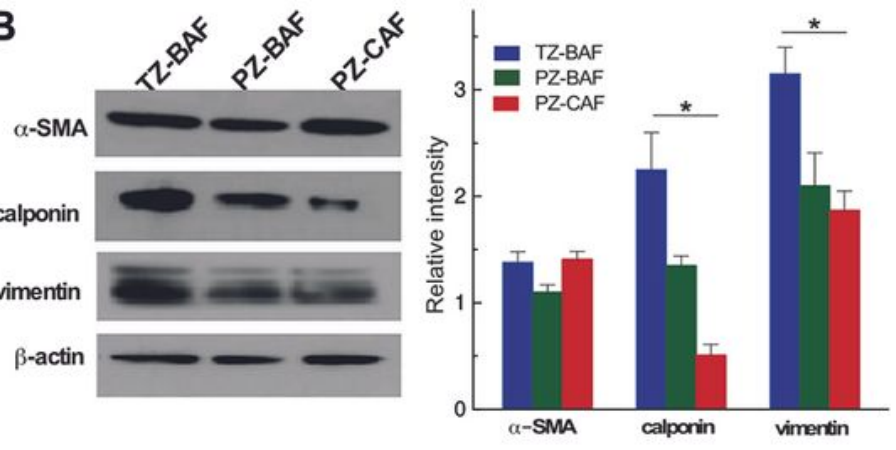

D

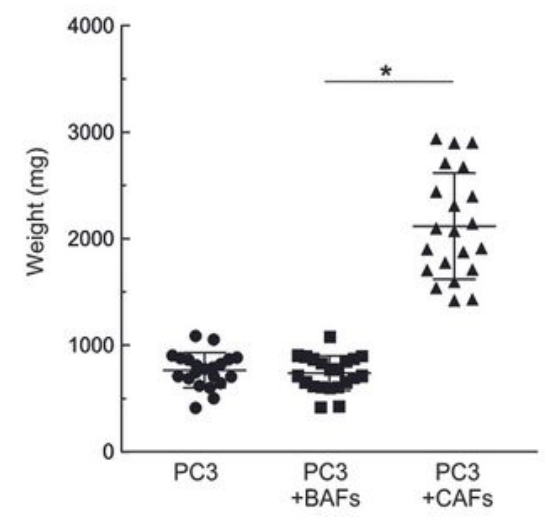

\section{Figure 4}

Phenotypic and functional stromal characterization of primary cultures of HPSCs isolated from benign or malignant human prostate tissue. A. mRNA expression of the activated-stroma markers were analyzed using RT-PCR in HPSCs from TZ-BAF, PZ-BAF, and PZ-CAF. Values are representative of averages \pm SEM, from 3 independent experiments, each one performed in triplicate. $B$. Western blot analyses of the activated-stroma markers aSMA, calponin and vimentin in primary cultures of BAF and CAF. C-D. In vivo 
effect on tumor growth of BAF and CAF using a PC3 cell line-derived xenograft model. C. Representative images of tumors obtained from each condition. D. Evaluation of tumor weight after tumor dissection. * $\mathrm{p}<0.01$.

\section{Supplementary Files}

This is a list of supplementary files associated with this preprint. Click to download.

- SupplementaryInformationCerdalnfanteet.al..pdf 